04,06, 19

\title{
Радиометрический метод контроля токов утечки в диэлектриках
}

\author{
Физико-технический институт им. А.Ф. Иоффре, \\ Санкт-Петербург, Россия \\ E-mail: gga_holo@mail.ru, g.sotnikova@mail.ioffe.ru \\ Поступила в Редакцию 9 февраля 2021 г. \\ В окончательной редакции 20 февраля 2021 г. \\ Принята к публикации 24 февраля 2021 г.
}

( Г.Ю. Сотникова, Г.А. Гаврилов, К.Л. Муратиков, Р.С. Пассет, Е.П. Смирнова

\begin{abstract}
Предложен новый экспериментальный метод регистрации токов утечки (токов сквозной проводимости) в диэлектриках, основанный на анализе динамики изменения температуры образца при воздействии электрического поля. Регистрация температурного отклика образца с высокой точностью и быстродействием осуществляется бесконтактным фотодиодным ИК-температурным сенсором. Возможности метода продемонстрированы на образцах керамики $0.65 \mathrm{PbFe}_{2 / 3} \mathrm{~W}_{1 / 3} \mathrm{O}_{3}-0.35 \mathrm{PbTiO}_{3}$ (PFW-PT), для которой получены экспериментальные зависимости проводимости в широком диапазоне температур от 20 до $110^{\circ} \mathrm{C}$.
\end{abstract}

Ключевые слова: ток утечки, температурный отклик, ИК-температурный сенсор.

DOI: 10.21883/FTT.2021.06.50930.024

\section{1. Введение}

При исследованиях диэлектрических материалов, особенно применительно к задачам электроники и измерительной техники, предполагается, среди других внешних факторов, циклическое воздействие сильных электрических полей. Наличие токов утечки (токов сквозной проводимости) в таких материалах, и связанное с ними выделения Джоулева тепла, является одним из основных механизмов их пробоя [1,2]. Очевидно, что поиск закономерностей возникновения и эволюции токов утечки в диэлектриках является актуальной задачей, определяющей надежность и долговечность элементов, создаваемых на их основе.

Особый интерес для практических применений представляют сегнетоэлектрики, обладающие одновременно и характеристиками классических диэлектриков и способностью к поляризации, что обуславливает их широкое применение для создания пьезо- и пироэлектрических элементов а также конденсаторов. Среди сегнетоэлектрических материалов, нашедших применение в электронике, особое место занимают составы близкие к морфотропной фазовой границе (МФГ), в частности, твердые растворы на основе сегнетоэлекриковрелаксоров, расположенные на МФГ. Они выделяются экстраординарными электромеханическими свойствами, такими как высокие пьезоэлектрические коэффициенты $\left(d_{33}>1500 \mathrm{pC} / \mathrm{N}\right)$ и коэффициенты электромеханической связи $\left(k_{33}>90 \%\right)[3,4]$ а также активно исследуются как перспективные электрокалорические материалы [5]. В тоже время электропроводность этих материалов остается практически не исследованной. Во многом это связано с неоднозначностью определения истинного значения тока проводимости материала при использовании стандартного метода измерения, основанного на контроле тока во внешней цепи источника напряже- ния [2]. В отличие от проводников, в электрических схемах, содержащих сегнетоэлектрические элементы, наблюдается значительное изменение тока со временем после момента приложения электрического поля вследствие уменьшения тока абсорбции, обусловленного наличием поляризации (релаксационной и миграционной) и перераспределением свободных зарядов в объеме, но не сопровождающееся сквозной проводимостью материала [6]. Только после окончания всех релаксационных процессов в образце устанавливается ток сквозной проводимости, величина которого и определяет проводимость диэлектрика. Очевидно, что для сегнетоэлектрических материалов времена релаксационных процессов неизвестны и могут меняться в широких пределах, особенно в области фазового перехода, когда происходит изменение структуры материала. Неопределенность времени установления истинных токов проводимости значительно „замедляет“ процесс измерений и может привести к существенным ошибкам при их количественной оценке.

В данной работе представлен экспериментальный метод измерения проводимости материалов, основанный на анализе их температурного отклика при воздействии постоянного электрического поля в виде ступенчатой функции. В качестве модельного объекта выбран твердый раствор-сегнетоэлектрик $0.65 \mathrm{PbFe}_{2 / 3} \mathrm{~W}_{1 / 3} \mathrm{O}_{3}-0.35 \mathrm{PbTiO}_{3}$ (PFW-PT), расположенный на МФГ [7,8].

\section{2. Обоснование метода}

Использование внешнего воздействия в виде ступенчатой функции является важнейшим инструментом теории измерений для определения параметров любой динамической системы. Образец исследуемого матери- 
ала может рассматриваться в качестве такой термодинамической системы, которая описывается уравнением состояния $P=P(E, T)$, где $P-$ поляризация, $E-$ напряженность электрического поля, $T-$ абсолютная температура [9].

При приложении к образцу электрического поля $E$ (напряжения $U$ ) наличие электропроводности в исследуемом материале приведет к протеканию через него тока $I(t)$, временной характер которого будет определяться зависимостью проводимости образца от изменения его состояния в течение всего времени приложения поля. $\mathrm{B}$ соответствии с законом Джоуля-Ленца, за время $d t$ в нем выделяется тепловая энергия $d Q(t)=U_{o} \cdot I(t) \cdot d t$, которая расходуется на повышение температуры образца $T(t)$ и на теплоотдачу через его поверхность в окружающее пространство.

Рассмотрим типичный для исследований образец сегнетоэлектрической керамики в виде диска толщиной $L \sim 0.3-1 \mathrm{~mm}$ и диаметром $D \sim 10 \mathrm{~mm}$ с электродами, нанесенными на его лицевые поверхности. Отводом тепла от боковой поверхности такого образца можно пренебречь, так как площадь боковой поверхности значительно меньше площади лицевых поверхностей. В этих условиях распределение температуры внутри образца описывается одномерным уравнением теплопроводности

$$
\rho C \frac{\partial T}{\partial t}=K \frac{\partial^{2} T}{\partial x^{2}}+Q(x, t)=K \frac{\partial^{2} T}{\partial x^{2}}+\frac{U(t) \cdot I(t)}{V},
$$

где $\rho$ - плотность материала образца, $C-$ удельная теплоемкость, $K$ - теплопроводность, $Q-$ количество подводимой теплоты, $U(t)$ - напряжение, $I(t)-$ сила тока, $V$ - объем образца.

Граничные условия, для образцов, лицевые поверхности которых расположены при $x=0$ и $x=L$ с учетом теплоотвода в окружающую среду, имеют вид

$$
\left.K \frac{\partial t}{\partial x}\right|_{x=0, L}=\left.H\left(t-t_{0}\right)\right|_{x=0, L,}
$$

где $H-$ коэффициент теплоотдачи образца, $T_{0}-$ температура окружающей среды.

Полученная задача теплопроводности является нестационарной. Динамика изменения температуры образца характеризуется двумя постоянными времени $\tau_{1}=\frac{L^{2}}{\kappa}$ $(\kappa-$ коэффициент температуропроводности материала, $L-$ толщина образца), $\tau_{2}=\frac{\rho c L}{2 H}$ и скоростью изменения тока через образец. Ее особенностью является слабое изменение тока на временах, сравнимых с $\tau_{1}$, при подаче на исследуемый образец постоянного напряжения $U_{0}$. В этом случае, при $t>\tau_{1}$ распределение температуры внутри образца можно считать практически однородным и температура поверхности образца, $T_{s}$, будет соответствовать температуре его объема, что позволяет получить решение уравнения (1) в довольно простом виде [10]. Для рассматриваемого класса материалов (диэлектрические керамики) и типичной толщины исследуемых образов (менее $1 \mathrm{~mm}$ ) расчетное значение $\tau_{1} \leq 1 s$.
С другой стороны, постоянная времени $\tau_{2}$ в условиях свободного конвективного теплообмена для рассматриваемых образцов оказывается достаточно большой (величина $H \sim 30 \mathrm{~W} /\left(\mathrm{m}^{2} \cdot \mathrm{K}\right)$, экспериментально полученная нами для керамических образцов с близкими параметрами в аналогичных условиях [9], дает расчетное значение $\tau_{2} \sim 40 \mathrm{~s}$ ) и наблюдаемое изменение тока через образец происходит за существенно меньшие времена. Тогда, интегрируя уравнение (1) по толщине образца и используя граничные условия (2), для температуры поверхности образца справедливо соотношение

$$
\begin{aligned}
T_{s}(t) & \cong T_{0}+\frac{1}{\rho C L} \int_{t_{1}}^{t} d t^{\prime} U\left(t^{\prime}\right) i\left(t^{\prime}\right) e^{-\frac{2 H}{\rho C L}\left(t-t^{\prime}\right)} \\
& \cong T_{0}+\frac{U_{0}}{\rho C L} e^{-\frac{2 H}{\rho C L}\left(t-t_{1}\right)} \int_{t_{1}}^{t} d t^{\prime} i\left(t^{\prime}\right),
\end{aligned}
$$

где $i(t)$ - плотность тока утечки, протекающего через образец, $t=t_{1}-$ момент времени начала приложения электрического поля.

При подаче импульса напряжения в момент $t_{1}$ и его выключения в момент $t_{2}$ полный цикл изменения температуры поверхности будет описываться уравнениями

$$
\begin{gathered}
T_{s}(t) \cong T_{0}+\frac{U_{0}}{\rho C L} e^{-\frac{2 H}{\rho C L}\left(t-t_{1}\right)} \int_{t_{1}}^{t} d t^{\prime} i\left(t^{\prime}\right), \quad \text { при } t_{1}<t \leq t_{2} \\
T_{S}(t) \cong T_{s}\left(t_{2}\right) e^{-\frac{2 H}{\rho C L}\left(t-t_{2}\right)}, \quad \text { при } t>t_{2},
\end{gathered}
$$

где $t_{1}$ - время начала подачи импульса напряжения величиной $U_{0}, t_{2}$ - время его окончания.

В этом случае величина плотности тока утечки при $t_{1}<t<t_{2}$ будет связана со скоростью изменения температуры выражением

$$
\frac{d T_{s}(t)}{d t} \cong \frac{U_{0} i(t)}{\rho C L} e^{-\frac{2 H}{\rho C L}\left(t-t_{1}\right)} .
$$

Если измерение температуры образца производится за время $t<\tau_{2}$, т.е. система близка к выполнению адиабатического условия, то экспоненту в выражении (5) можно считать слабо изменяющейся $\left(e^{-\frac{2 H}{\rho L}\left(t-t_{1}\right)}\right) \cong 1$ и для определения тока утечки использовать соотношение

$$
\frac{d T_{s}(t)}{d t} \cong \frac{U_{0} i(t)}{\rho C L}=\frac{U_{0} I(t)}{m C},
$$

где $m=\rho L S$ - масса образца, $S$ - площадь его лицевых поверхностей, $I(t)=i(t) \cdot S$.

Из выражений (5) и (6) видно, что анализ динамики изменения температуры поверхности образца позволяет не только рассчитать величину тока проводимости при известных значениях его теплоемкости и массо-габаритных параметров, но и определить 
его функциональную зависимость, которая, при условии $U(t)=$ const $=U_{o}$, определяется только изменением проводимости образца и представляет собой неявную функцию температуры $I(T(t))$. В общем случае величина коэффициента теплоотдачи $H$ является важным фактором при использовании решений уравнения теплопроводности (1) с граничными условиями (2) в виде (5) или (6). Ранее нами была предложена методика определения коэффициента теплоотдачи твердотельных объектов методом фототермической ИК-радиометрии [10], которая хорошо согласуется с предлагаемым методом измерения токов утечки и была использована в наших экспериментах.

\section{3. Экспериментальные результаты}

На рис. 1 представлена экспериментальная зависимость изменения температуры образца керамики PFW-PT при подаче на него ступенчатого импульса напряжения.

Образцы представляли собой диски диаметром 8 и толщиной $0.82 \mathrm{~mm}$. На лицевые поверхности образцов нанесены Ag электроды, покрытые поглощающим веществом для повышения излучательной способности образца, что необходимо для повышения точности измерения температуры объекта при использовании радиометрических методов. Непрерывное измерение температуры образца осуществлялось бесконтактным фотодиодным ИК-температурным сенсором, на основе фотодиода. чувствительного в области среднего ИК-диапазона спектра $4.2 \pm 0.2 \mu \mathrm{m}$ (MIR-сенсор, от английского middle range infrared sensor), в составе многоканальной измерительной установки, подробно рассмотренной в работе [9], и ссылки в ней. Точность измерения температуры MIR-сенсором с временным откликом $2 \mathrm{~ms}$ составляет \pm 0.025 градуса при $20^{\circ} \mathrm{C}$ и увеличивается до \pm 0.005 градуса при температурах выше $100^{\circ} \mathrm{C}$ при сохранении быстродействия. Масса образца (с электродами и поглощающим слоем) составляла $423.5 \mathrm{mg}$. $\mathrm{B}$ качестве источника постоянного напряжения использовался усилитель высокого напряжения TREK 609E-6.

Из рис. 1 видно, что при электрическом воздействии в форме ступенчатого импульса напряжения амплитудой $800 \mathrm{~V}$ и длительностью $15 \mathrm{~s} \quad\left(t_{1}=22 \mathrm{~s}-\right.$ время начала, $t_{2}=37 \mathrm{~s}-$ время окончания импульса) образец нагревается до температуры выше $120^{\circ} \mathrm{C}$, a затем медленно остывает с постоянной времени $\tau \sim 30$ s. Экспоненциальный характер нагрева образца указывает на наличие в нем значительных токов утечки, которые возрастают с повышением его температуры. Аппроксимация температурной зависимости остывания образца экспоненциальной функцией вида (4) при $t>t_{2}$ позволяет экспериментально определить показатель экспоненты $2 H / \rho C L$ и рассчитать значение коэффициента теплоотдачи $H$. Для исследуемых образцов при удельной теплоемкости $C=330 \mathrm{~J} /(\mathrm{kg} \cdot \mathrm{K})$

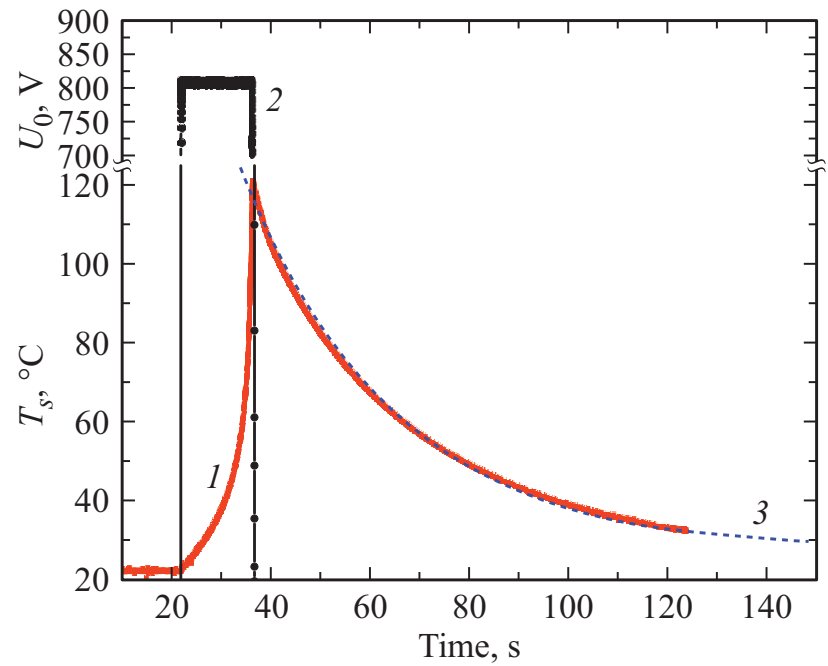

Рис. 1. Экспериментальные зависимость изменения температуры (линия 1) керамики PFW-PT при подаче ступенчатого импульса напряжения (линия 2) амплитудой $800 \mathrm{~V}$ и длительностью $15 \mathrm{~s}$. Пунктирная линия (3) - аппроксимация температуры остывания образца экспоненциальной функцией с постоянной времени $\tau=32.2 \mathrm{~s}$.

(типичное значение для керамических материалов) было получено значение $H=38.75 \mathrm{~W} /\left(\mathrm{m}^{2} \cdot \mathrm{K}\right)$. Полученное значение $H$ позволяет оценить справедливость применения приближенного выражения (6) для расчета тока утечки по экспериментальной температурной зависимости нагрева образца. Типичные для керамических материалов значения величин теплоемкости и теплопроводности $(1.32 \mathrm{~W} /(\mathrm{m} \cdot \mathrm{K}),[9])$ и экспериментально полученные значений толщины $(L=0.82 \mathrm{~mm})$ и плотности $\left(\rho=7680 \mathrm{~kg} / \mathrm{m}^{3}\right)$ образца материала, позволяют рассчитать значения постоянных времени $\tau_{1}=0.9 \mathrm{~s}$ и $\tau_{2}=27 \mathrm{~s}$, характеризующих динамику изменения температуры образца (см. раздел 2), и использовать решение уравнения теплопроводности в виде (6) для расчета тока утечки в течение всего времени воздействия импульса напряжения длительностью $15 \mathrm{~s}$, начиная с первой секунды после его начала.

Таким образом, численное дифференцирование экспериментального температурного отклика образца на ступенчатое воздействие электрического поля позволяет рассчитать величину тока утечки. При этом, методическая ошибка измерений зависит только от точности определения массы образца и справедливости предположения о постоянстве величин теплоемкости материала С в исследуемом диапазоне температур. Хорошее совпадение экспоненциальной аппроксимирующей функции (пунктирная линия на рис. 1) с экспериментальной температурной кривой остывания образца свидетельствует о справедливости предположения о несущественном влиянии изменения теплоемкости $C$. Чувствительность метода ограничена точностью регистрации температуры и определяется параметрами образца $(m, C)$ а также 
величиной приложенного напряжения $U_{0}$ и допустимой длительностью импульса напряжения, не приводящими к перегреву и пробою. Для образцов, рассмотренных в данной работе, при амплитуде импульса $U_{0}=800 \mathrm{~V}$, чувствительность составляет $0.15 \mu \mathrm{A}$ при регистрации температурного отклика сенсором с быстродействием $2 \mathrm{~ms}$ и может быть повышена до $4 \mathrm{nA}$ при увеличении времени измерения до $1 \mathrm{~s}$.

Результат расчета величины тока утечки для керамики PFW-PT представлен на рис. 2 (линия 1). На этом же рисунке показан результат мониторинга тока в цепи высоковольтного источника напряжения (линия 2), который хорошо совпадает с расчетной зависимостью, полученной в результате тепловых измерений.

Полученная зависимость тока утечки в течение длительного воздействия постоянным напряжением, сопровождающимся значительным нагревом образца, позволила рассчитать температурную зависимость его удельной проводимости. Текущие значения удельной проводимости образца $\gamma(t)$ рассчитывались в соответствии с выражением $i(t)=\gamma t \cdot E$, где $i-$ плотность тока, $E-$ напряженность электрического поля. Измеряемыми величинами в нашем эксперименте являлись напряжение $U_{0}$, прикладываемое к образцу, и временная зависимость температуры образца $T(t)$, которая связана с величиной плотности тока утечки через свою производную (6). Это позволяет записать выражение для расчета удельной проводимости образца через измеряемые величины

$$
\frac{d T}{d t}=\frac{U_{0} \cdot \gamma(t) \cdot U_{0} / L}{\rho C L}=\gamma(t) \cdot \frac{U_{0}^{2}}{\rho C L^{2}} .
$$

На рис. 3 (линия 1) приведена зависимость удельной проводимости сегнетоэлектрической керамики PFW-PT. Скорость изменения температуры образца $d T / d t$, была получена методом численного дифференцирования из экспериментальной зависимости $T(t)$ с временным разрешением $2 \mathrm{~ms}$, представленной на рис. 1 (линия 1 ).

На рис. 3 также представлена зависимость величины приложенного напряжения, соответствующая текущей температуре образца, для которой проводился расчет величины удельной проводимости материала (линия 2). Как видно из рисунка, удельная проводимость сегнетоэлектрика PFW-PT возрастает от $\gamma=0.04 \mu \mathrm{S} / \mathrm{m}$ при температуре $22^{\circ} \mathrm{C}$ до $\gamma=22 \mu \mathrm{S} / \mathrm{m}$ при температуре $109^{\circ} \mathrm{C}$, соответствующей температуре образца при окончании воздействия поля. Наблюдаемый дальнейший рост температуры образца (и величины удельной проводимости) связан с временной задержкой при снятии напряжения, однако, полученные количественные значения нельзя считать истинными, так как они не удовлетворяют условию, при котором справедливо выражение (6).

Для верификации предложенного радиометрического метода измерения токов утечки и расчета проводимости, керамика PFW-PT была исследована на измерительной установке Easy Check 300 [ACCT Systems GmbH, Aachen Germany], широко используемой для исследования петель поляризации и токов утечки сегнетоэлектриков.

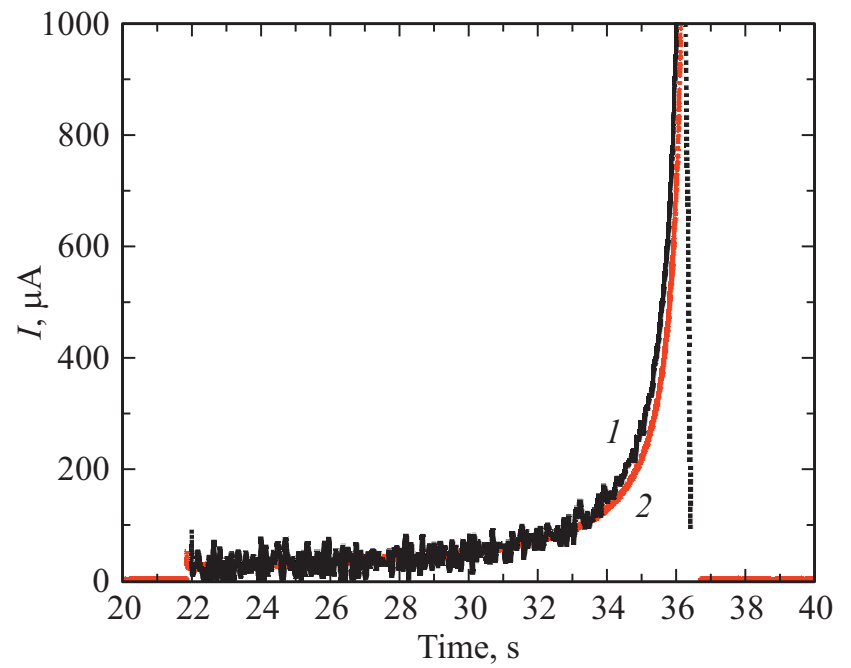

Рис. 2. Расчетная зависимость тока утечки керамики PFW-PT (линия 1) в соответствии с выражением (6) и экспериментальная зависимость (линия 2) тока в цепи высоковольтного источника напряжения.

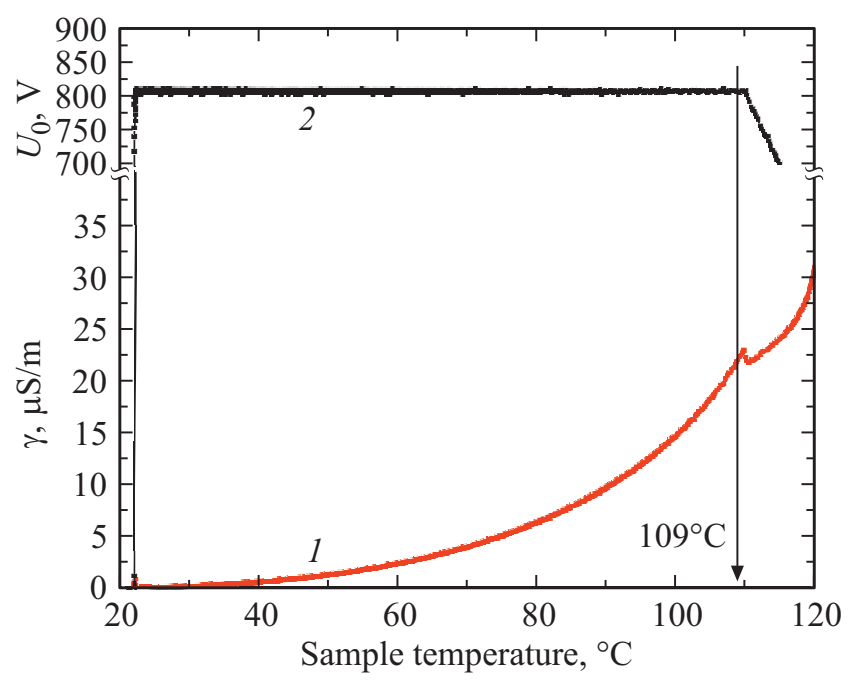

Рис. 3. Температурная зависимость удельной проводимости керамики PFW-PT (линия 1). Линия 2 - приложенное напряжение, соответствующее текущей температуре образца.

На рис. 4 представлена зависимость плотности тока утечки керамики PFW-PT, полученная на измерительной установке EasyCheck при напряжении характерном для таких измерений $U_{\max }=3 \mathrm{~V}$.

Следует заметить, что при увеличении напряжения и длительности времени измерения на EasyCheck возможен неконтролируемый разогрев образца и полученный результат не может считаться достоверным. Расчетное значение удельной проводимости при комнатной температуре $\left(\sim 22^{\circ} \mathrm{C}\right)$ из данных рис. 4 соответствует $\gamma=0.036 \mu \mathrm{S} / \mathrm{m}$. Эта величина хорошо совпадает со значением $\gamma=0.04 \mu \mathrm{S} / \mathrm{m}$, полученным в эксперименте в результате анализа температурного отклика на сту- 


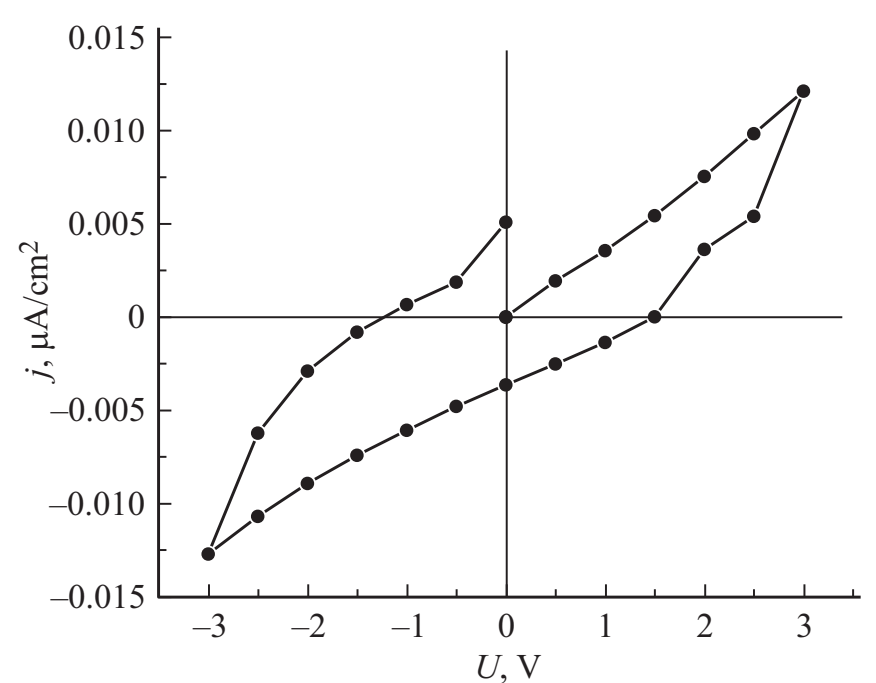

Pис. 4. Зависимость плотности тока утечки керамики PFW-PT, полученная на измерительной установке EasyCheck при $U_{\max }=3 \mathrm{~V}$.

пенчатое электрическое воздействие амплитудой $800 \mathrm{~V}$. При этом следует особо отметить, что в соответствии с методикой измерения токов утечки на Easy Check, время измерения величины проводимости материала только для одной температурной точки при $U_{\max }=3 \mathrm{~V}$ составило $6 \mathrm{~s}$ (для $10 \mathrm{~V}$, соответственно, $20 \mathrm{~s}$ ), в то время как с помощью предложенного нами метода за $12 \mathrm{~s}$ была получена информация о зависимости проводимости материала в широком диапазоне температур от 20 до $110^{\circ} \mathrm{C}$.

\section{4. Заключение}

Предложенный радиометрический метод контроля проводимости диэлектриков сочетает в себе возможность визуализации токов утечки с количественной оценкой их истинной величины при экспериментальных исследованиях и применениях материалов в сильных электрических полях. Метод основан на анализе температурного отклика образца на воздействие электрическим полем, который определяется исключительно токами сквозной проводимости. В случае сегнетоэлектриков, метод не чувствителен к наличию неконтролируемых по времени токов абсорбции, ограничивающих применение методов, основанных на контроле тока во внешней цепи источника напряжения.

Измерительные преимущества предложенного метода обусловлены использованием фотодиодных ИК-температурных сенсоров и применением базовых принципов теории измерений параметров термодинамических систем. Анализ теплообмена образца с окружающей средой является определяющим при расчете истинного значения тока проводимости материала.

Предложенный экспериментальный метод позволяет оперативно получить данные о температурной зависи- мости проводимости материалов и тем самым оптимизировать характер воздействия электрического поля для обеспечения их эффективной работы в безопасном режиме, не приводящем к перегреву и последующему пробою. При этом время измерения температурной зависимости проводимости значительно сокращается по сравнению с традиционным методом измерений.

Впервые получены экспериментальные данные о температурной зависимости удельной проводимости сегнетоэлектрика $0.65 \mathrm{PbFe}_{2 / 3} \mathrm{~W}_{1 / 3} \mathrm{O}_{3}-0.35 \mathrm{PbTiO}_{3}$, которая увеличивается более чем в 500 раз при изменении температуры от 20 до $110^{\circ} \mathrm{C}$.

\section{Конфликт интересов}

Авторы заявляют, что у них нет конфликта интересов.

\section{Список литературы}

[1] Г.А. Воробьев, Ю.П. Похолков, Ю.Д. Королев, В.И. Меркулов. Физика диэлектриков (область сильных полей). Томский политехнический ун-т. (2011). 245 с.

[2] Н.П. Богородицкий, В.В. Пасынков, Б.М. Тареев. Электротехнические материалы. Энергоатомиздат. Л. (1985). $304 \mathrm{c}$.

[3] S. Zhang, F. Li. J. Appl. Phys. 111, 3, 031301 (2012).

[4] S. Zhang, F. Li, J. Luo, R. Sahul. IEEE Transact. Ultrasonics, Ferroelectrics Frequency Control 60, 1572 (2013).

[5] J. Perantie, H.N. Tailor, J. Hagberg, H. Jantunen, Z.-G. Ye. J. Appl. Phys, 114, 17, 174105 (2013).

[6] К. Окадзаки. Технология керамических диэлектриков, Энергия, М, 1976.

[7] L. Feng, Z.-G. Ye. J. Sol. State Chem. 163, 2, 484 (2002).

[8] G.A. Gavrilov, G.Y. Sotnikova, A.V. Sotnikov, E.P. Smirnova. J. Mater. Sci., 55, 16, 6783 (2020).

[9] G.Yu. Sotnikova, G.A. Gavrilov, A.A. Kapralov, K.L. Muratikov, E.P. Smirnova. Rev. Sci. Instrum. 91, 1, 015119 (2020).

[10] С.Е. Александров, Г.А. Гаврилов, А.А. Капралов, К.Л. Муратиков, Г.Ю. Сотникова. ПЖТФ 43, 14, 104 (2017).

Редактор Т.Н. Василевская 